\title{
Investigation of Effect of Pore Shape, Position and Passivation on the Thermoelectric Properties of Porous Armchair Silicene Nanoribbons
}

\author{
Rajneesh Kumari ${ }^{1}$, Deep Kamal Kaur Randhawa ${ }^{2}$ and Sukhdeep Kaur ${ }^{3 *}$ \\ ${ }^{1 \& 3}$ Department of Electronics Technology, Guru Nanak Dev University, Amritsar, Punjab, India \\ ${ }^{2}$ Department of Electronics and Communication Engineering, Guru Nanak Dev University, RC Jalandhar, Punjab, India \\ *Corresponding Author \\ E-Mail: rk.angurala@gmail.com, randhawadk@gmail.com, gndusukhdeep@gmail.com
}

\begin{abstract}
In this paper, thermoelectric properties of porous armchair silicene nanoribbons (ASiNRs) have been investigated as a function of pore shape, position and passivation using the Non-equilibrium Green's function (NEGF) method and Extended Huckel Technique (EHT). Here, nanopores of circular, rectangular, rhombus and triangular nature at different positions have been incorporated with an intention to optimize the structure for maximum thermoelectric figure of merit. In addition, the effect of passivation of the pore edges on the thermoelectric performance has been studied for all the shapes. Further, the effect of temperature variation on the thermoelectric efficiency has been studied. Ballistic transport regime and semi-empirical method using Huckel basis set are used to obtain the electrical properties, while the Brenner potential is used for the phononic system.
\end{abstract}

Keywords: Pore, Nanoribbon, Thermoelectric, Figure of Merit, Thermal Conductivity

\section{INTRODUCTION}

Silicene is a two-dimensional (2D) one-atom-thick crystalline form of silicon, one of the most abundantly and widely used materials in the electronics industry [1]. The major driving force behind these materials lies in the fact that, by engineering their atomic structure we can open an energy band gap in these materials $[3,4]$.

Silicene is being studied with an effort to understand its thermoelectric properties and enhance the thermoelectric performance with an intention of designing silicon compatible electronic devices to utilize thermoelectric energy. Silicon in bulk has high thermal conductance which is less desirable for thermoelectric conversion. One promising route is to take advantage of reduced phonon thermal conductance in low dimensional materials which can be obtained by nanostructuring it to create nanoribbons $[1,5,6]$.

Thermal conductance reduction could be further engineered in porous silicene nanoribbons (ASiNRs) since phonon thermal conductance is reduced in nanoporous semiconductors $[1,7]$. Considering the fact that carbon materials are not compatible with the present microelectromechanical systems (MEMS) technology, the possibility of replacing conventional MEMS technology based on bulk silicon with silicon-based nanotechnology will draw the attention of the electronics industry toward ASiNRs since such a material is promising for prolonging the validity of Moore's Law [4].

In recent times, the scientific interest have been attracted towards challenge of removing heat from nanoelectronic devices and thermoelectrically converting waste heat into electricity [1]. Additionally, ASiNR is slightly buckled structure, which opens up the possibility of a tunable band gap [8] which is essential for a better value of Seebeck coefficient. The efficiency of a thermoelectric device and material is determined by its thermoelectric figure of merit (ZT) defined as:

$$
Z T=\frac{S^{2} G T}{k}
$$

Where, $\mathrm{S}$ is the Seebeck coefficient (thermopower), $\mathrm{G}$ is the electrical conductance, $\mathrm{T}$ the temperature and $\mathrm{k}$ the thermal conductance given by $\mathrm{K}=\mathrm{Ke}+\mathrm{Kph}$, where $\mathrm{Ke}(\mathrm{Kph})$ is the electronic (phononic) contribution to $\mathrm{k}$. ZT could be enhanced by increasing the power factor $\left(S^{2} G\right)$ or decreasing the thermal conductance and therefore a highperformance thermoelectric material should possess a large Seebeck coefficient and electrical conductance and simultaneously a low thermal conductance [1]. However, these factors are correlated and thus require a delicate optimization to yield higher ZT values [5].

Both theoretical predictions and experimental demonstrations have shown that in comparison to the bulk materials low-dimensional or nanoscale systems could exhibit much higher ZT values, which leads to a very important prospect of thermoelectric applications [10]. Sadeghi et al., [1] have proposed the structure of nanoporous ASiNRs with varying edge termination and concluded that the proper tuning of the Fermi energy can significantly improve the electronic thermoelectric figure of merit. Pan et al ., [10] have theoretically studied the thermoelectric performance of pristine armchair and zigzag SiNRs and predicted that by optimizing the doping level, width, and applied temperature, the ZT value of SiNRs could be enhanced to 4.9 .

Also, ASiNRs exhibit higher ZT values than zigzag SiNRs at room and higher temperatures. Kaur et al., [7] have analyzed the effect of various pore dimensions on the 
thermoelectric performance of SiNR-NP devices and concluded that using the optimal values for the pore dimensions, value of ZT could be attained 3 times higher than that of ASiNR without a pore.

\section{MODELING AND COMPUTATIONAL METHOD}

In the present work, enhancement of thermoelectric performance of ASiNRs with introduction and passivation of nanopores (NPs) has been studied. Nanoporous armchair ASiNR with optimized buckling and lattice constant $0.53 \AA$ and 3.6, respectively [7]. The width $W r$ and length $L r$ of the ribbon are $4.07 \mathrm{~nm}$ (21 atoms wide) and $7.42 \mathrm{~nm}$, respectively, while the pore position has been varied to achieve superior performance. By varying the number of atoms in the adjacent edges, which in turn is a measure of pore width, electrical conductivity could be improved with a significant reduction in the thermal conductance. These parameters in turn enhance the thermoelectric performance. We consider electrons transferring Ballistically through the system assuming that electron-phonon scattering is reduced.

Within the Extended Huckel theory framework, $G^{R}=\left(E S_{o v^{-}}\right.$ $\left.H-\Sigma_{L^{-}}-\Sigma_{R}\right)^{-1}$ is the retarded Green function where $E$ is the electron energy, $H$ and $S_{o v}$ are the Hamiltonian and overlap matrix of the central region respectively, which are calculated from extended Huckel technique using Hoffman basis [11,12]. Once the retarded Green's function is obtained, the electron transmission function can be obtained through [13]:

$$
T(E)=\operatorname{Trace}\left[\Gamma_{R}(E) G^{R}(E) \Gamma_{L}(E) G^{R \dagger}(E)\right]
$$

Where, $\Gamma_{L, R}(E)=i\left(\Sigma_{L, R}(E)-\Sigma_{L, R}(E)\right)$ describes the level broadening due to the coupling between the left / right electrodes and the central scattering region and $\Sigma_{L, R}(E)$ are the retarded self-energies for the electrodes. The mesh points in real space calculation are $1 \times 1 \times 100 \mathrm{k}$ points, mesh cut-off at 10 Hartree and the temperature was set at $300 \mathrm{~K}$.

Once $T(E)$ is known, Seebeck Coefficient $S$, electrical conductance $G$ and electronic thermal conductance $k_{e}$ can be obtained under linear response approximation as [14]:

$$
\begin{aligned}
& G=\left.\frac{I}{\Delta V}\right|_{\Delta T=0}=e^{2} L_{0} \\
& S=-\left.\frac{\Delta V}{\Delta T}\right|_{I=0}=\frac{L_{1}}{e T L_{0}} \\
& k_{e}=\left.\frac{I_{Q}}{\Delta T}\right|_{I E=0}=\frac{L_{2}-L_{1}^{2} / L_{0}}{T}
\end{aligned}
$$

where,

$$
L_{n}=\frac{2}{h} \int d E T(E)\left(-\frac{\partial f}{\partial E}\right)(E-\mu)^{n}
$$

The formalism used here is from the work of Esfarjani et al., [15] Here, $L_{n}$ is Lorenz function, $e$ is the charge of electron, $h$ is the Planck constant, $\mu$ is the chemical potential, $T$ is the average temperature and $\partial f / \partial E$ is the derivative of the Fermi function $\left.\left(f=1+\exp \left((E-\mu) / k_{B} T\right)\right)^{-1}\right)$, [16] known as Fermi-Dirac distribution function. To calculate the phonon thermal conductance, we used the Landauer approach [14]

$$
K_{p h}=\frac{h^{2}}{2 \pi k_{B} T^{2}} \int d \omega \omega^{2} T_{p h}(\omega)\left(\frac{\partial n}{\partial \omega}\right)
$$

For phonon transmission $T_{p h}(\omega), H$ and $S$ are substituted by a force constant matrix $\mathrm{K}$, and a diagonal matrix of atomic mass $M$ respectively. The term $\partial n / \partial \omega$ is the derivative of the Bose-Einstein distribution for phonons. To calculate the force constant matrix for phonons, empirical Brenner potential (Brener_CH_2005) was used. Both the electron and phonon transport simulations are performed in the Virtual NanoLab Atomistix Toolkit (ATK) software package [17]. Each structure is optimized before transport calculations with its atom coordinates relaxed so that the forces on individual atoms are minimized to be smaller than $0.01 \mathrm{eVÁ}-1$.

\section{RESULTS AND DISCUSSION}

Firstly, the thermoelectric performance of ASiNRs embedded with different pore positions corresponding to center and the respective edges (left and right) of the nanoribbon have been calculated at temperature $300 \mathrm{~K}$. Further the effect of increasing the nanopores from single to double has been predicted. In the later section, the change in thermoelectric performance due to pore passivation and temperature dependence is also studied.

A. Circular Pore: The atomistic model of armchair Silicene Nanoribbon structures with circular pores is shown in Fig.1. For the calculation of thermoelectric figure of merit, the pore size is kept constant at a diameter of $1.552 \mathrm{~nm}$. As it shows each pore has its own associated edge terminations with two channels on both sides of the nanoribbon. It is observed that in case of single pore, position has weak dependence on ZT $(\mathrm{ZT}=0.1469)$, while in double porous case, the value of ZT becomes almost three times greater than single porous case i.e. 0.3808 . The variation in values of parameters $S, G, K_{p h}$ and $Z T$ are plotted with respect to chemical potential of the nanopore. For the peak value of $\mathrm{ZT}$, at the same chemical potential $(-0.11 \mathrm{eV})$ Seebeck coefficient and conductance $204.6 \mu \mathrm{V} / \mathrm{K}$ and $4.498 * 10^{-5}$ respectively, are obtained.

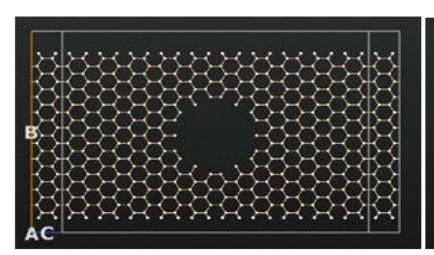

(a)

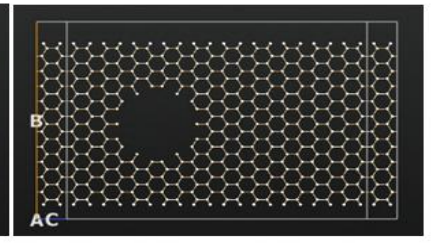

(b)

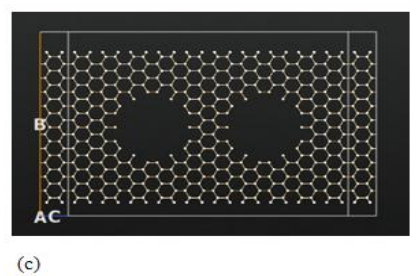

(c)

Fig. 1 Geometrical optimized structure of the Armchair Silicene nanoribbon with circular nanopore thermoelectric device with pore diameter $1.552 \mathrm{~nm}$ 


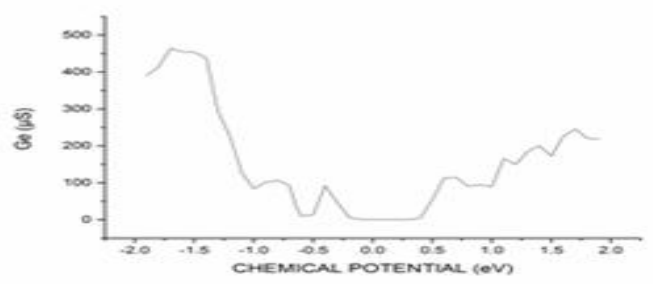

(a)

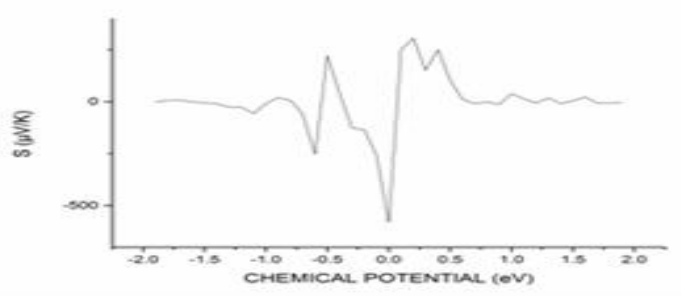

(c)

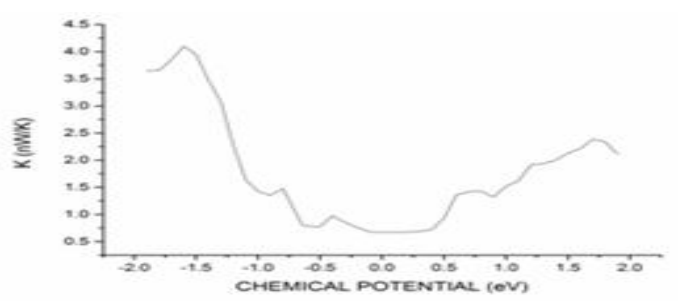

(b)

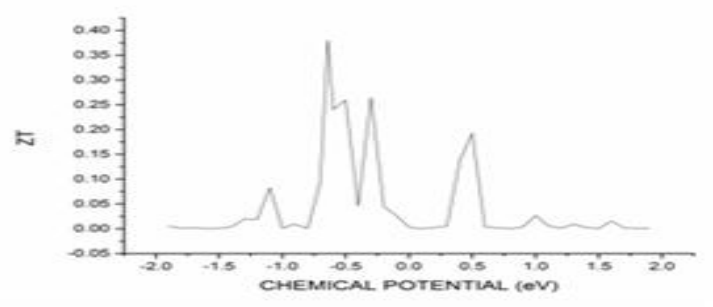

(d)

Fig. 2 (a) Conductance, (b) Seebeck Coefficient, (c) Thermal conductance and (d) figure of merit for the circular nanopore ASiNR

B. Rectangular Pore: The atomistic model of our proposed armchair Silicene Nanoribbon structures with rectangular pores are shown in Fig. 3. As it shows each pore has its own associated edge terminations with two channels on both sides of the nanoribbon. The length and width of the pore is kept constant as $2.25 \mathrm{~nm}$ and $1.552 \mathrm{~nm}$, respectively. It is observed that in case of single pore, position has weak dependence on ZT $(\mathrm{ZT}=0.1096)$, while in double porous case, the value of $\mathrm{ZT}$ becomes greater than single porous case i.e. 0.1591 . The variation in values of parameters $S, G$, $\mathrm{K}_{\mathrm{ph}}$ and ZT are plotted with respect to chemical potential of the nanopore. The highest Seebeck coefficient obtained is $70.6 \mu \mathrm{V} / \mathrm{K}$ at -0.72 , while the highest conductance is $9.845^{*} 10^{-5}$. The two pore rectangular shape Armchair SiNR shows the optimum results.

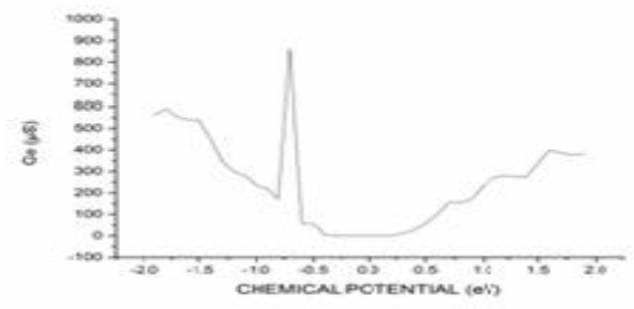

(a)

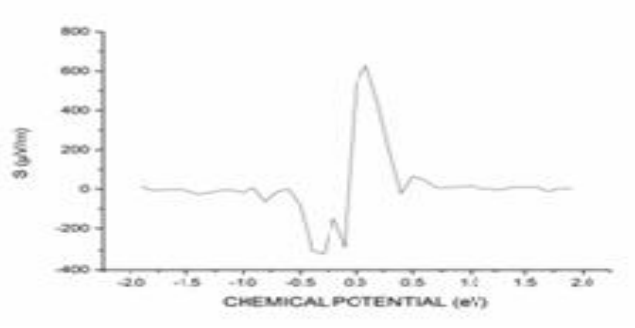

(e)

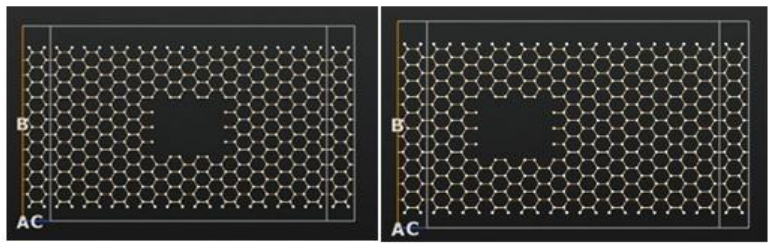

(a)

(b)

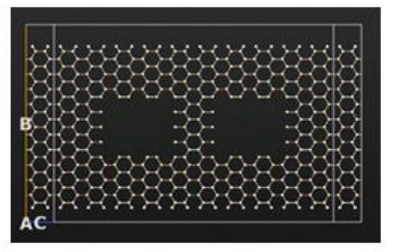

(c)

Fig. 3 Geometrical optimized structure of the Armchair Silicene nanoribbon with rectangular nanopore thermoelectric device

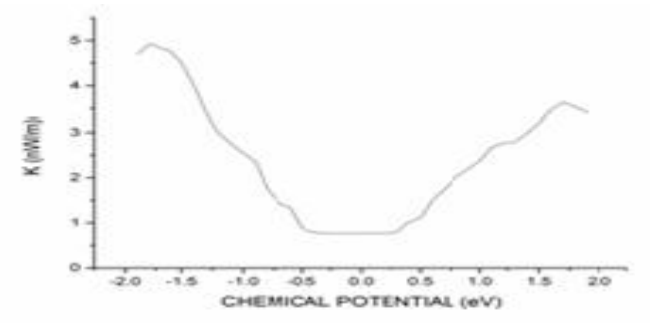

(b)

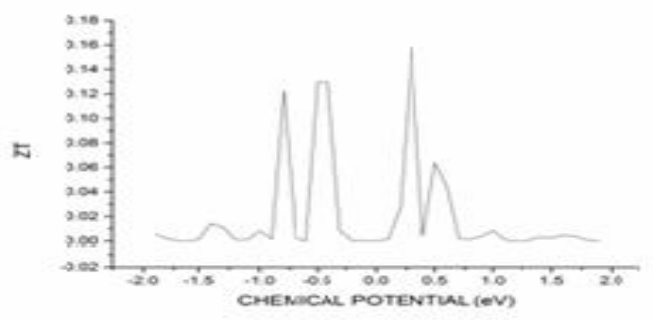

(d)

Fig. 4 (a) Conductance, (b) Seebeck Coefficient, (c) Thermal conductance and (d) figure of merit for the rectangular nanopore ASiNR 
C. Rhombus Pore: The atomistic model of our proposed armchair Silicene nanoribbon structure with rhombus pores is shown in Fig. (5). As it shows each pore has its own associated edge terminations with two channels on both sides of the nanoribbon. The side of Rhombus pore is kept at $1.746 \mathrm{~nm}$. It is observed that in case of single pore, position has weak dependence on ZT $(Z T=0.2953)$, while in double porous case, the value of ZT drops slightly to $\mathrm{ZT}=$ 0.2873 . The variation in values of parameters $\mathrm{S}, \mathrm{G}, \mathrm{K}_{\mathrm{ph}}$ and ZT are plotted with respect to chemical potential of the nanopore. The highest Seebeck coefficient obtained is 184.2 $\mu \mathrm{V} / \mathrm{K}$ at -0.34 , while the highest conductance is $2.68 * 10^{-}$ ${ }^{5}$.The thermal conductivity is reduced significantly, which in turn leads to the enhancement of figure of merit. The double pore rhombus ASiNRs show the best result among the other pore positions.

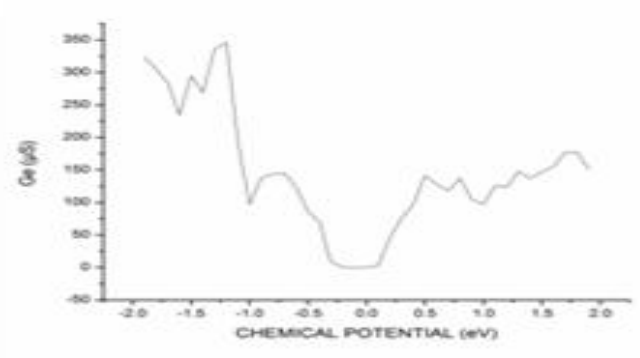

(a)

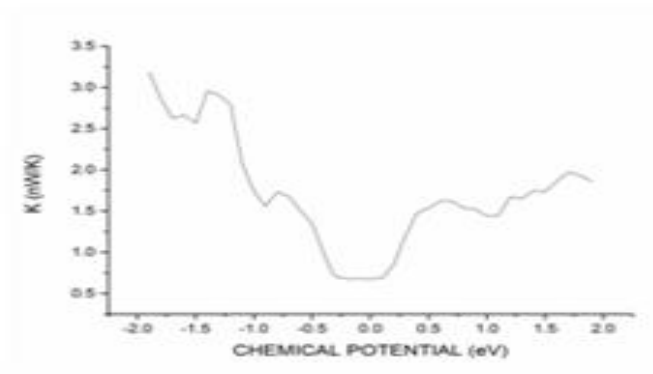

(c)
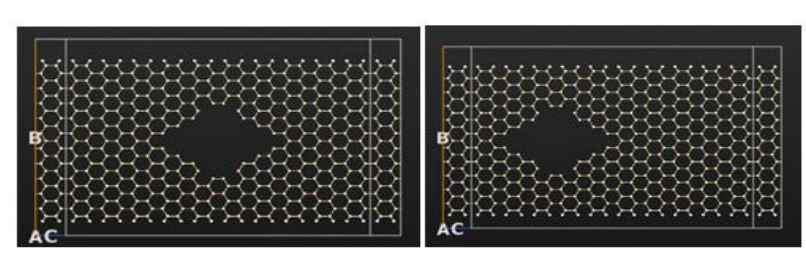

(a)

(b)

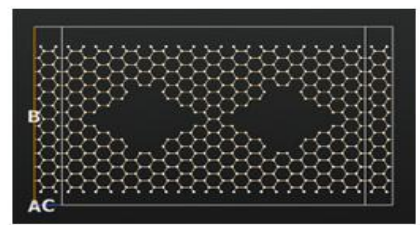

(c)

Fig. 5 Geometrical optimized structure of the Armchair Silicene nanoribbon with rhombus nanopore thermoelectric device

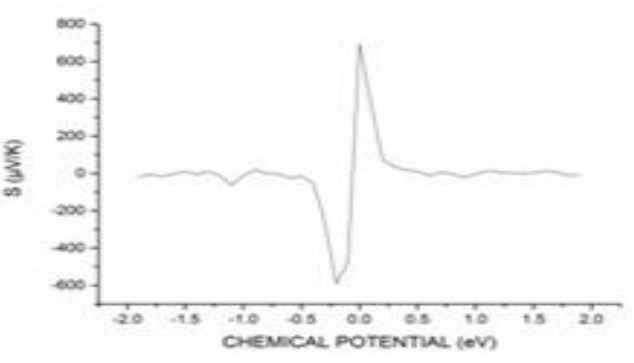

(b)

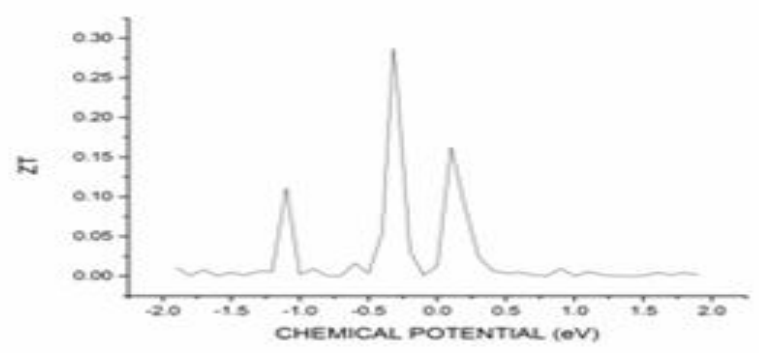

(d)

Fig. 6 (a) Conductance, (b) Seebeck Coefficient, (c) Thermal conductance and (d) figure of merit for the rhombus nanopore ASiNR

D. Triangular Pore: The atomistic model of our proposed armchair Silicene ribbon structures with triangular pores is shown in Fig (7). As it shows each pore has its own associated edge terminations with two channels on both sides of the nanoribbon. The side of the triangle is kept at $1.94 \mathrm{~nm}$. The symmetry of the pores is maintained in each case. It is observed that in case of single pore, position has weak dependence on $\mathrm{ZT}(\mathrm{ZT}=0.1794)$, while in double porous case, the value of $\mathrm{ZT}$ is greater than single porous case i.e. $\mathrm{ZT}=0.3002$. The variation in values of parameters $\mathrm{S}, \mathrm{G}, \mathrm{K}_{\mathrm{ph}}$ and $\mathrm{ZT}$ are plotted with respect to chemical potential of the nanopore. The highest Seebeck coefficient obtained is $209.3 \mu \mathrm{V} / \mathrm{K}$ at -0.72 , while the highest conductance is $3.961 * 10^{-5}$. The two pore triangular ASiNR structure shows the best result among the other pores.
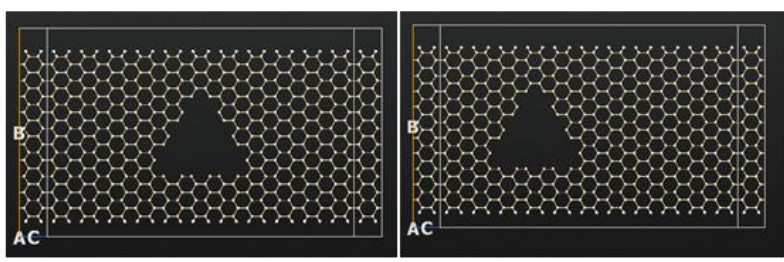

(a)

(b)

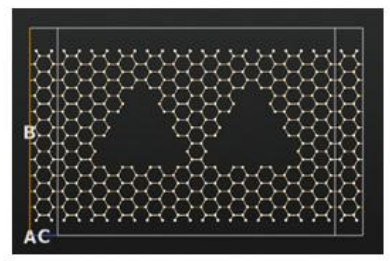

(c)

Fig. 7 Geometrical optimized structure of the Armchair Silicene nanoribbon with triangular nanopore thermoelectric device 


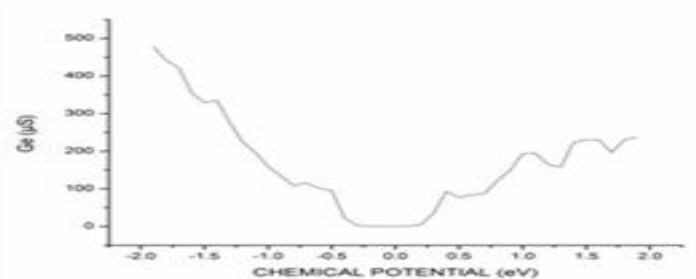

(a)

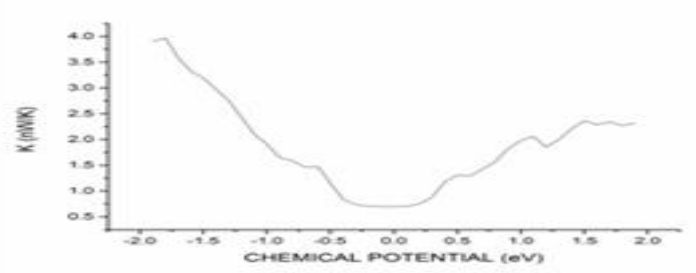

(c)

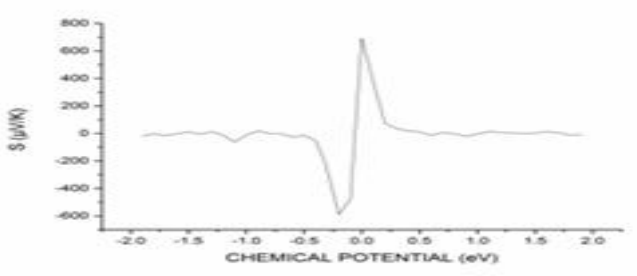

(b)

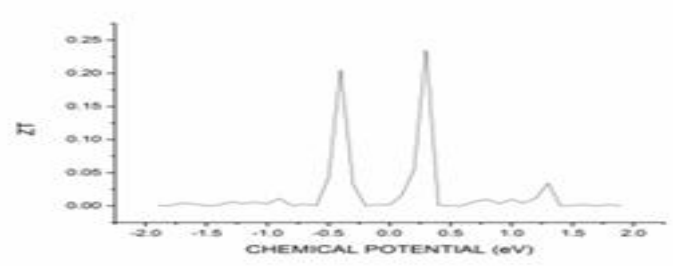

(d)

Fig. 8 (a) Conductance, (b) Seebeck Coefficient, (c) Thermal conductance and (d) figure of merit for the triangular nanopore ASiNR

E. Comparison of All Pore Shapes: To examine the dependence of thermoelectric performance of nanostructure on the shape and position of the pore, the results obtained from all the pore shapes and their respective positions have been compared. Fig 9 illustrates a comparative analysis among the peak observed ZT for different cases. It is analyzed that in the case of single pore nanoribbon (centre and left pore as shown in Fig.9) the figure of merit of individual pore shape is not severely affected by when the pore position changed. Moreover, the rhombus nanopore structure shows the maximum figure of merit followed by triangular pore, circular and rectangular pore shape being the last. The order of the figure of merit is:

\section{RHOMBUS $>$ TRIANGULAR $>$ CIRCULAR $>$ RECTANGULAR}

However, thermoelectric performance is enhanced with addition of another pore as shown as in the fig. for two pore structure. In case of double pore the peak ZT value is exhibited by circular pore followed by rhombus shape, triangular shape and rectangular one being the last. It can be predicted from the same fig. that the position of the pore (left, centre, right) has slight dependence on the thermoelectric parameters. ZT of circular pore increases from 0.1469 in single pore case to 0.3808 for the double pore.

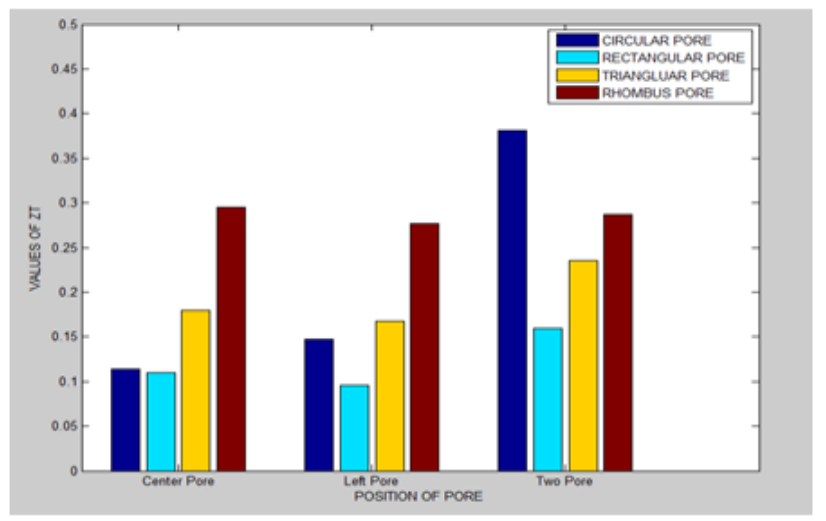

Fig. 9 comparison of the figure of merit for all the pore shapes and pore position

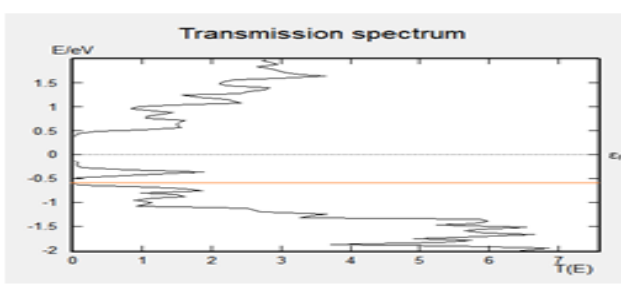

(a)

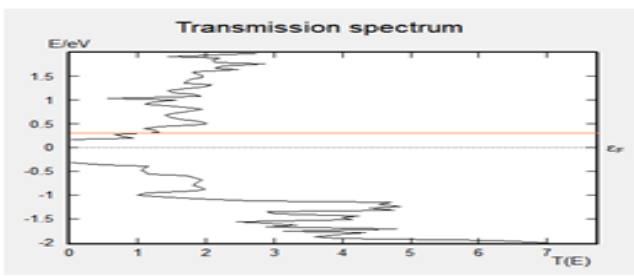

(c)

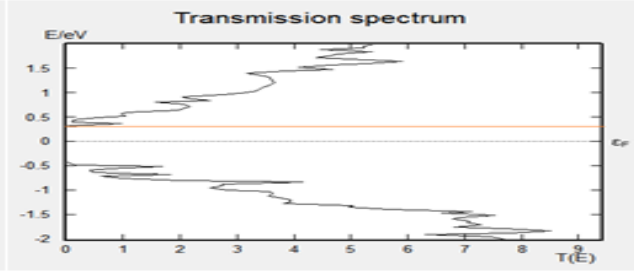

(b)

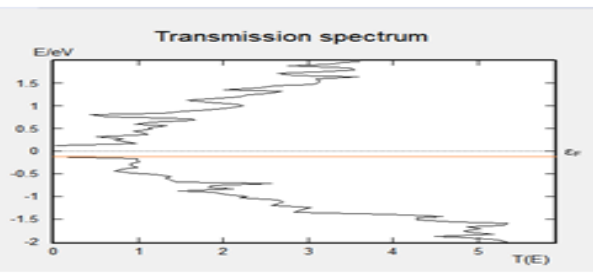

(d)

Fig.10 Transmission spectrum of double porous SiNRs for (a) circular, (b) rectangular, (c) rhombus and (d) triangular nanopores with orange line representing the chemical potential, where highest ZT obtained 


\section{PORE PASSIVATION}

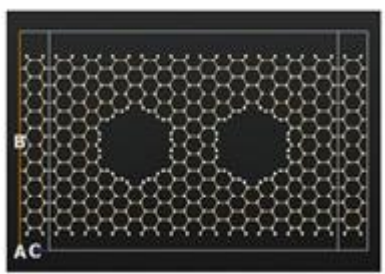

(a)

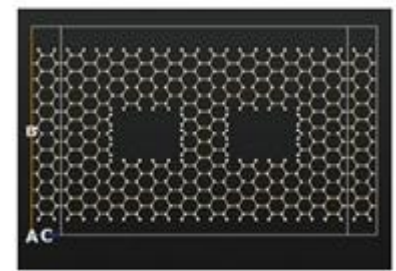

(c)

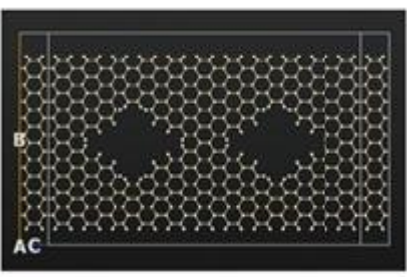

(b)

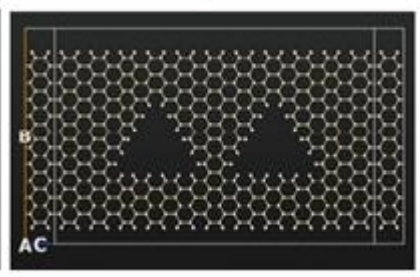

(d)

Fig. 11 Geometrical optimized structure of the Armchair Silicene nanoribbon with passivated nanopore of all pore shapes thermoelectric device

In addition, the influence of passivation of the pore edges on the performance of the thermoelectric properties of ASiNRs has been analyzed. Fig. 12 (a-d) compares the results obtained for passivated pore edges for all shapes are then compared with the non- passivated results obtained so far.

As observed for the Fig. 12 (a, c-d), the effects of pore passivation are not much pronounced since there is very

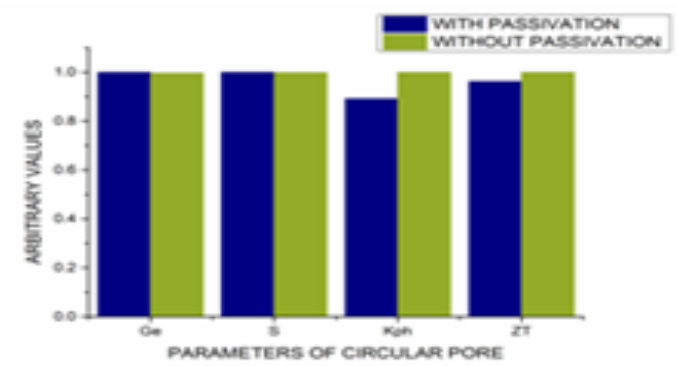

(a)

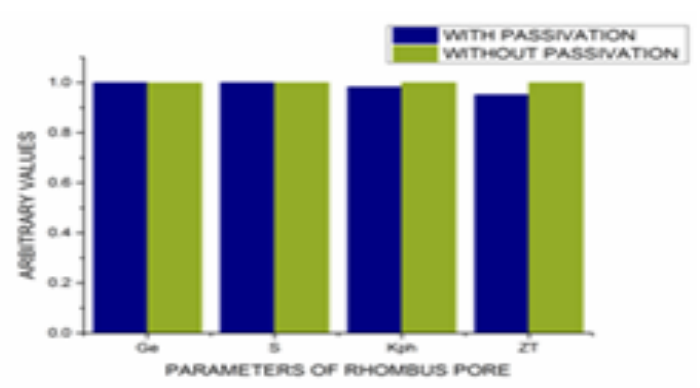

(c) little or no effect on the thermoelectric properties. However, from Fig.12 (b), it is analyzed that the thermoelectric figure of merit for rectangular pore has been enhanced significantly, which is almost twice the value of $\mathrm{ZT}$ for rectangular pore in passivated case.

\section{TEMPERATURE DEPENDENCE}

Fig.13 illustrates the effect of temperature dependence on the thermoelectric properties of single porous Silicene Nano structure (Rectangular in this case). It is seen that the temperature affects the figure of merit greatly. At low temperatures of $150 \mathrm{~K}$, lower values of figure of merit are observed. But a sudden increase in the value of $\mathrm{ZT}$ is noticed for intermediate temperature as it reaches its highest value extending till 900K. However, for higher temperatures, the value of ZT starts decreasing afterwards.

The power factor $\left(\mathrm{S}^{2} \mathrm{G}\right)$ decreases abruptly till $150 \mathrm{~K}$ and then it starts increasing steadily till $900 \mathrm{~K}$. The value of $K_{e}$ increases steadily up to $500 \mathrm{~K}$ and thereafter it rises sharply up to its highest value. At the same time, $K p h$ increases gradually and after $500 \mathrm{~K}$ it becomes stable. The sum of $\mathrm{K}_{\mathrm{e}}$ and $\mathrm{K}_{\mathrm{ph}}$ equates to the $\mathrm{K}$ which denotes the thermal conductivity. We need low thermal conductivity and higher power factor to achieve higher figure of merit. To achieve a better figure of merit delicate optimization of these factors should be done as these factors are correlated.

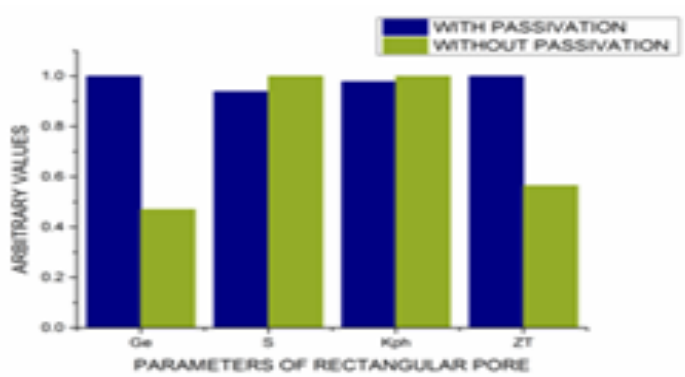

(b)

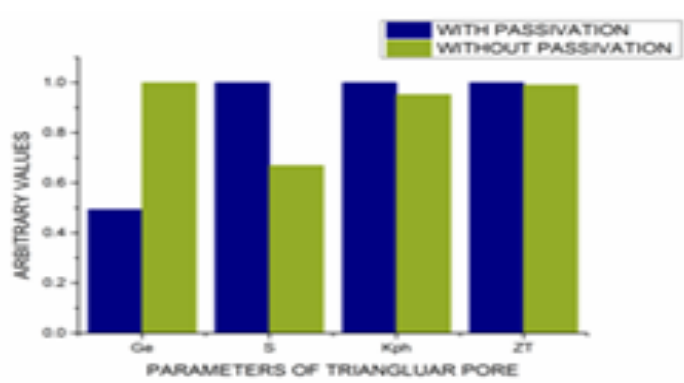

(d)

Fig. 12 comparison for various parameters of passivated and non-passivated pores for (a) circular pore, (b) rectangular pore, (c) rhombus pore and (d) triangular pore 


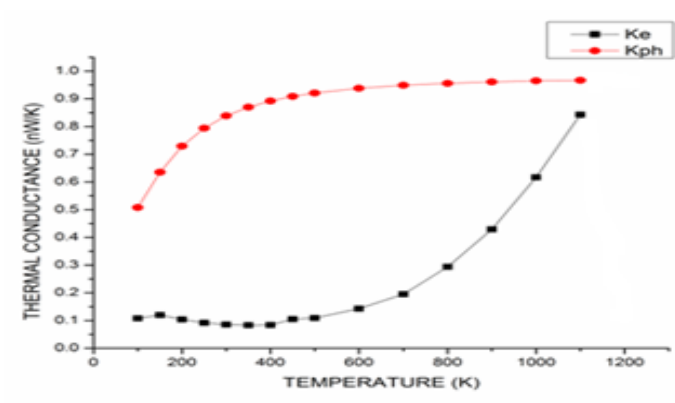

(a)

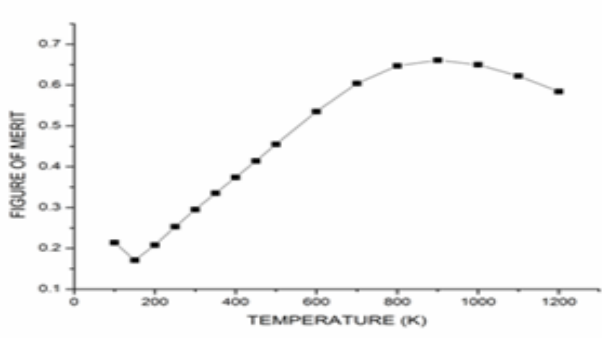

(b)

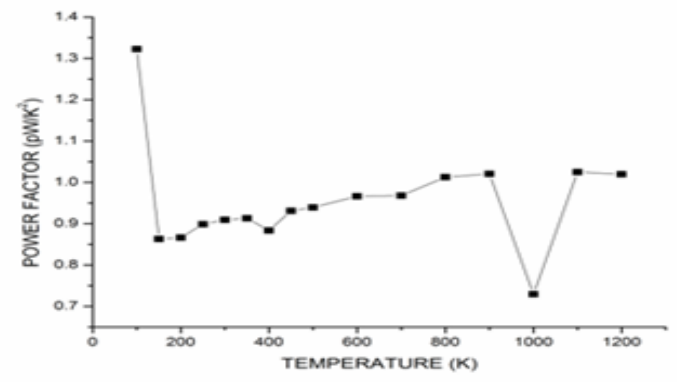

(c)

Fig.13 temperature dependence of (a) Thermal conductance, (b) Power Factor and (c) figure of merit for the rectangular single pore ASiNR structure

\section{CONCLUSION}

In summary, we have investigated the influence of different pore shapes and its associated position on the thermoelectric performance of ASiNR-NP devices. Nanopores of different shapes i.e. circular, rectangular, triangular and rhombus have been introduced at varying positions of the nano structure so as to study their effect on the thermoelectric figure of merit. By optimizing the pore parameters, we have been able to achieve higher Figure of merit, which is approximately three times higher than pristine ASiNR. Further, the effect of pore passivation is observed, which has been successfully able to enhance ZT in case of Rectangular pores. Thus, our work extends the idea towards achieving better thermoelectric devices by optimal pore efficiency.

\section{REFERENCES}

[1] H. Sadeghi, S. Sangtarash, and C.J. Lambert, "Enhanced thermoelectric efficiency of porous silicene nanoribbons", Sci. Rep. Vol. 5, 9514, March 2015.

[2] Y. Liang, V. Wang, H. Mizuseki, and Y. Kawazoe, "Band gap engineering of silicene zigzag nanoribbons with perpendicular electric fields: a theoretical study", J. Phys. Condens. Matter Vol. 24, 455302, Oct. 2012.

[3] Y.L. Song, Y. Zhang, J.M. Zhang, D.B. Lu, and K.W. Xu, "Modulation of the electronic and magnetic properties of the silicene nanoribbons by a single C chain.", Eur. Phys. J. B Vol. 79, No. 2, pp. 197-202, 2011.

[4] C. Le'andri, G. Lay, B. Aufray, C. Girardeaux, J. Avila, M. E. Davila, M. C.Asensio, C. Ottaviani, and A. Cricenti, "SiGe, Ge and related compounds 6: materials, processing and devices, ECS Transactions", Vol. 64, 2005.

[5] M.S. Hossain, F.A. Dirini, F.M. Hossain, and E. Skafidas, "High performance Silicenenano-ribbon thermoelectric devices by incorporation and dimensional tuning of nanopores", Sci. Rep. Vol. 5, 11297, June 2015.
[6] L.D. Hicks and M.S. Dresselhaus, "Thermoelectric figure of merit of a one dimensional conductor", Phys. Rev. B, Vol. 47, pp. 1663116634, June 1993.

[7] S. Kaur, D.K. Randhawa, and S.B. Narang, "Theoretical investigation of effect of pore size and porepassivation on the thermoelectric performance of silicene nanoribbons", Vol. 32, pp. 3485-3493, Sept. 2017.

[8] Z. Ni, H. Zhong, X. Jiang, R. Quhe, Y. Wang, J. Yang, J. Shi, and J. $\mathrm{Lu}$, "Tunable band gap and doping type in silicene by surface adsorption: Towards tunneling transistors", Nanoscale Vol. 6, pp. 7609-7618, April 2014.

[9] Y. Qi, Z. Wang, M. Zhang, F. Yang, and X. Wang, "Thermoelectric devices based on one dimensional nanostructures", J. Mater. Chem. A Vol. 1, pp. 6110-6124, Feb. 2013.

[10] L. Pan, H. J. Liu, X. J. Tan, H. Y. Lv, J. Shi, X. F. Tang and G. Zheng, "Thermoelectric properties of armchair and zigzag silicene nanoribbons", Phys. Chem. Chem. Phys., Vol. 14, pp. 13588-13593, Aug. 2012.

[11] D. Kienle, J.I. Cerda, and A.W. Ghosh, "Extended Huckel theory for band structure, chemistry, and transport. Carbon nanotubes", J. Appl. Phys. Vol. 100, 043714, Aug. 2006.

[12] R. Landauer, "Spatial variation of currents and fields due to localized scatterers in metallicconduction", IBM J. Res. Dev. Vol. 1, pp. 223231, July 1957.

[13] M.S. Hossain, F. Al-Dirini, F.M. Hossain, and E. Skafidas, "High performance Silicenenano-ribbon thermoelectric devices by incorporation and dimensional tuning of nanopores", Sci. Rep. Vol. 5, 11297, June 2015.

[14] G.Q. Zhang, Q.X. Yu, W. Wang, and X.G. Li, "Nanostructures for thermoelectric applications, synthesis, growth mechanism \& property studies", Adv. Mater. Vol. 22, pp. 1959-1962, April 2010.

[15] K. Esfarjani, M. Zebarjadi, and Y. Kawazoe, "Thermoelectric properties of a nanocontact made of two-capped single-wall carbon nanotubes calculated within the tight binding approximation", Phys. Rev. B: Condens. Matter Mater. Phys. Vol. 73, 085406, Feb. 2006.

[16] H. Sadeghi, S. Sangtarash, and C.J. Lambert, "Enhancing the thermoelectric figure of merit in engineered graphene nanoribbons", Beilstein J. Nanotechnol. Vol. 6, pp. 1176-1182, May 2015.

[17] Atomistic Tool Kit Manual, Quantum wise Inc. Atomistix toolkit version 13.8.0, Quantum wise $\mathrm{A} / \mathrm{S}$ [Online] Available: http://quantumwise.com/ 